\title{
Performance Evaluation of Closed-Loop Spatial Multiplexing Codebook Based on Indoor MIMO Channel Measurement
}

\author{
Junjun Gao, Jianhua Zhang, Yanwei Xiong, Yanliang Sun, and Xiaofeng Tao \\ Key Laboratory of Universal Wireless Communications of Ministry of Education, Beijing University of Posts and Telecommunications, \\ Mailbox 92, Beijing 100876, China \\ Correspondence should be addressed to Junjun Gao, gaojunjun@bupt.edu.cn
}

Received 23 July 2012; Revised 24 October 2012; Accepted 24 October 2012

Academic Editor: Ai Bo

Copyright ( $(2012$ Junjun Gao et al. This is an open access article distributed under the Creative Commons Attribution License, which permits unrestricted use, distribution, and reproduction in any medium, provided the original work is properly cited.

\begin{abstract}
Closed-loop MIMO technique standardized in LTE can support different layer transmissions through precoding operation to match the channel multiplexing capability. However, the performance of the limited size codebook still needs to be evaluated in real channel environment for further insights. Based on the wideband MIMO channel measurement in a typical indoor scenario, capacity loss (CL) of the limited size codebook relative to perfect precoding is studied first in two extreme channel conditions. The results show that current codebook design for single layer transmission is nearly capacity lossless, and the CL will increase with the number of transmitted layers. Furthermore, the capacity improvement of better codebook selection criterions is very limited compared to CL. Then we define the maximum capacity boost achieved by frequency domain layer adaption (FDLA) and investigate its sensitivity to SNR and channel condition. To survey the effect of frequency domain channel variation on MIMOOFDM system, we define a function to measure the fluctuation levels of the key channel metrics within a subband and reveal the inherent relationship between them. Finally, a capacity floor resulted as the feedback interval increases in frequency domain.
\end{abstract}

\section{Introduction}

The rapid data traffic growth in indoor environment raises a great challenge to modern communications to meet the peak data rate requirement. Thanks to the spatial parallel transmission, MIMO multiplexing technique can achieve promising spectrum efficiency and attracts large interests recently [1-4]. In general, better performance can be achieved if the channel state information (CSI) is available at the transmitter [5]. Fortunately, the indoor MIMO channel is usually slow time-varying due to the low mobility of the mobile terminals and scatters, which provides the favorable condition for applying closed-loop MIMO scheme to feed the CSI back. However, it is unrealistic to transmit the full CSI back to the transmitter due to the feedback channel limitation. Standardized in LTE, the transmitter and receiver share a common precoding matrix table (codebook), also only rank indicator (RI) and precoding matrix index (PMI) are sent back to indicate the number of transmitted layers and corresponding precoding matrix [6].
Codebook-based MIMO precoding systems have attracted great research interests recently. Channel capacity with quantized precoding matrix is studied in independent and identically distributed channels [7], where the precoding matrix is selected from a random codebook. Overloaded vector precoding [8] is proposed for single-user MIMO channels, where the number of data streams is larger than the minimum number of the transmit and receive antennas. The codebook-based precoding and equalization are jointly designed to improve the performance [9]. Codebook-based lattice-reduction-aided precoding is studied for coded MIMO systems to reduce the amount of feedback [10]. However, the limited size codebook usually results in capacity loss $(\mathrm{CL})$ as a result of the interlayer interference $[10,11]$. From the perspective of codebook selection, minimum CL is equivalent to maximize the capacity, yet no literature has studied it to reduce the complexity. Some recent indoor channel measurement results focus on different points, such as eigenvalue distribution [12], multilink separation [13], and dense multipath characteristics [14]. Performance evaluation is 
important for practical MIMO deployment, such as HSDPA [15] and E-UTRA [16]. However, the precoding performance has not been evaluated in real indoor channel environment.

Increasing the system bandwidth (e.g., up to $100 \mathrm{MHz}$ ) is another effective method to satisfy the high data rate requirement. Consequently, the channel frequency-selective characteristic expands the available adaption dimension in MIMOOFDM systems. Frequency domain layer adaption (FDLA) in subband level [6] can enhance the average spectral efficiency and achieve a tradeoff between the performance and amount of feedback bits. However, the performance of FDLA is greatly influenced by the channel variation within a subband. We would like to know the maximum performance potential of FDLA in real frequency-selective channels, and its relationship to the channel condition and SNR. The frequency domain variation of the eigenvalues is theoretically studied in adaptive MIMO beamforming systems [17]. The effect of frequency domain variation on the MIMO precoding performance has not been investigated in real indoor channels.

Channel measurement is the most straightforward and reliable method to acquire the real channel characteristics [18]. Based on the wideband MIMO channel measurement in a typical indoor scenario at $6 \mathrm{GHz}$, we propose a metric to distinguish various MIMO channel conditions. Two extreme cases of our campaign are selected for performance evaluation, which yield poor and fine multiplexing capabilities, respectively. In this paper, we focus on the following key problems.

(i) How is the CL related to channel condition, receiver type, and the number of transmitted layers?

(ii) Does the CL provide some insight into the codebook selection? Compared to the CL caused by the limited feedback, how much capacity improvement can be achieved by the use of better codebook selection criterion?

(iii) What is the maximum capacity boost of FDLA? How is it related to the channel condition and SNR?

(iv) How to measure the fluctuation levels of the key channel metrics within a subband? Is there any inherent relationship between them?

The major contributions of this paper are organized as follows. The exact CL expression and its upper bound are derived for single-layer and full-rank transmissions. We first utilize the concept of CL to obtain low-complexity codebook selection criterion, which can reduce the interlayer or interuser interference for single-user or multiuser MIMO. A robust channel condition metric is defined based on the measured channel data analysis. The maximum performance potential of FDLA in real frequency-selective channels is studied, and its relationship to the channel condition and SNR is also investigated. We define a function to measure the key channel metric fluctuation level within a subband and investigate the effect of frequency domain variation on MIMO-OFDM systems. Based on the proposed methods, the precoding performance is systematically evaluated in real indoor channel environment.
The remainder of this paper is organized as follows. Section 2 introduces the system model and proposes the framework of performance evaluation. Section 3 describes the measurement equipment and environment. Section 4 presents the key simulation results. In Section 5, a summary of this paper is given.

Notation. The $(i, j)$ th element of the matrix $\mathbf{A}$ is $\{\mathbf{A}\}_{i, j}$. The superscript $T$ and $\dagger$ represent the transpose and Hermitian transpose, respectively. The symbol $\operatorname{tr}(\cdot)$ denotes the matrix trace, and $\|\cdot\|_{F}^{2}$ is the square of the matrix Frobenius norm. The notation $\mathcal{E}(\cdot)$ is the expectation operation.

\section{System Model and Evaluation}

2.1. MIMO Precoding System Model. Considering a narrowband MIMO system with $N_{t}$ transmit and $N_{r}$ receive antennas, the channel matrix is denoted as $\mathbf{H} \in \mathcal{C}^{N_{r} \times N_{t}}$. Assuming $N_{t} \leq N_{r}$, the received signal can be represented as

$$
\mathbf{y}=\mathbf{H x}+\mathbf{n},
$$

where $\mathbf{y} \in \mathcal{C}^{N_{r} \times 1}$ is the received vector, $\mathbf{x} \in \mathcal{C}^{N_{t} \times 1}$ is the transmitted vector, and $\mathbf{n} \in \mathcal{C}^{N_{r} \times 1}$ is the additive noise vector with independent and identical distributed random elements satisfying $\mathcal{C} \mathcal{N}\left(0, \sigma^{2}\right)$.

For fully exploiting the space dimension flexibility of the multiple antenna system, precoding operation is performed to achieve different layers transmission. Supposing the number of transmitted layers is $L$, the precoding process is expressed as $\mathbf{x}=\mathbf{P s}$, where $\mathbf{P} \in \mathcal{C}^{N_{t} \times L}$ is the unitary precoding matrix satisfying $\mathbf{P}^{\dagger} \mathbf{P}=\mathbf{I}_{L}$. The effective $L \times 1$ transmit symbol vector is $\mathbf{s}=\left[s_{1}, \ldots, s_{L}\right]^{T}$, and the received signal can be rewritten as [5]

$$
\mathbf{y}=\text { HPs }+\mathbf{n} .
$$

Assuming that the total transmit power $P_{T}$ is uniformly distributed to $L$ layers, $P_{T}=\mathcal{E}\left(\mathbf{s}^{\dagger} \mathbf{s}\right)=L \mathcal{E}\left(\left|s_{i}\right|^{2}\right)$, so the received SNR is $\gamma_{0}=P_{T} / \sigma^{2}$.

Linear receiver can be used to recover the symbol of each layer with low complexity, and we consider zero-forcing (ZF) and minimum mean square error (MMSE) receivers in this paper. The output of the linear receiver can be represented as $\widehat{\mathbf{y}}=\Psi \mathbf{y}$, and the corresponding linear combiner $\Psi$ and the SINR of the $l$ th layer are, respectively, $[11,19]$

$$
\begin{gathered}
\Psi_{\mathrm{ZF}}=\left(\mathbf{P}^{\dagger} \mathbf{H}^{\dagger} \mathbf{H P}\right)^{-1}(\mathbf{H P})^{\dagger}, \\
\operatorname{SINR}_{l}=\frac{\gamma_{0}}{L} \frac{1}{\left\{\left(\mathbf{P}^{\dagger} \mathbf{H}^{\dagger} \mathbf{H} \mathbf{P}\right)^{-1}\right\}_{l, l}}, \\
\boldsymbol{\Psi}_{\mathrm{MMSE}}=\left(\frac{L}{\gamma_{0}} \mathbf{I}+\mathbf{P}^{\dagger} \mathbf{H}^{\dagger} \mathbf{H} \mathbf{P}\right)^{-1}(\mathbf{H P})^{\dagger}, \\
\operatorname{SINR}_{l}=\frac{1}{\left\{\left(\mathbf{I}+\frac{\gamma_{0}}{L} \mathbf{P}^{\dagger} \mathbf{H}^{\dagger} \mathbf{H} \mathbf{P}\right)^{-1}\right\}_{l, l}}-1 .
\end{gathered}
$$


The total capacity of $L$ layers transmission with ZF and MMSE receivers can be represented as [11]

$$
C_{L}=\sum_{l=1}^{L} \log _{2}\left(1+\operatorname{SINR}_{l}\right)
$$

The singular value decomposition (SVD) of $\mathbf{H}^{\dagger} \mathbf{H}$ is $\mathbf{H}^{\dagger} \mathbf{H}=\mathbf{V} \boldsymbol{\Lambda} \mathbf{V}^{\dagger}$, where the matrix $\mathbf{V}$ is unitary. The ordered diagonal elements of $\boldsymbol{\Lambda}$ are $\lambda_{\max }=\lambda_{1} \geq \cdots \geq \lambda_{N_{t}}=$ $\lambda_{\min } \geq 0$, and $\lambda_{l}$ is the $l$ th eigenvalue of $\mathbf{H}^{\dagger} \mathbf{H}$. For $L$ layers transmission, the perfect precoding matrix $\mathbf{P}=\mathbf{V}_{L}$ is the first $L$ columns of $\mathbf{V}, \mathbf{V}_{L}=\left[\mathbf{v}_{1}, \ldots, \mathbf{v}_{L}\right]$, where $\mathbf{v}_{k}$ denotes the $k$ th column of $\mathbf{V}$.

Under perfect precoding assumption, parallel and independent layers are constructed as a result of the interlayer interference elimination. Hence, ZF and MMSE receivers are the same for any given SNR, then (4) becomes

$$
\left.C_{L}\right|_{\mathbf{P}=\mathbf{V}_{L}}=\sum_{l=1}^{L} \log _{2}\left(1+\frac{\gamma_{0}}{L} \lambda_{l}\right) .
$$

Theorem 1. In the high SNR region, the single layer transmission will outperform the full-rank transmission, $C_{1} \geq C_{N_{t}}$, if the channel matrix $\mathbf{H}$ satisfies the following inequality:

$$
\|\mathbf{H}\|_{F}^{2}-\lambda_{\max } \leq \frac{1}{\gamma_{0}} N_{t}\left(N_{t}-1\right)\left(N_{t}^{1 /\left(N_{t}-1\right)}-1\right) .
$$

Proof. In the high SNR region, from (4), we have

$$
\begin{aligned}
C_{N_{t}} \approx & \log _{2}\left(\frac{\gamma_{0}}{N_{t}} \lambda_{1}\right)+\sum_{l=2}^{N_{t}} \log _{2}\left(1+\frac{\gamma_{0}}{N_{t}} \lambda_{l}\right) \\
\leq & \left(N_{t}-1\right) \log _{2}\left(1+\frac{\gamma_{0}}{N_{t}\left(N_{t}-1\right)} \sum_{l=2}^{N_{t}} \lambda_{l}\right) \\
& +\log _{2}\left(\frac{\gamma_{0}}{N_{t}} \lambda_{1}\right) .
\end{aligned}
$$

The proof can be easily completed using the property of the matrix trace and Frobenius norm.

For the special case of $2 \times 2 \mathrm{MIMO}$ configuration, (6) is simplified to

$$
\lambda_{\min } \leq \frac{2}{\gamma_{0}} .
$$

It indicates that the Demmel condition number $K_{D}$ should satisfy the following term:

$$
K_{D}=\frac{\|\mathbf{H}\|_{F}^{2}}{\lambda_{\min }} \geq \frac{\gamma_{0}\|\mathbf{H}\|_{F}^{2}}{2}
$$

and the channel matrix will be more ill-conditioned as the SNR increases.
2.2. Codebook Capacity Loss. In practice, it is impossible for the transmitter to obtain the perfect precoding matrix due to the limitation of the feedback channel capacity. The RI and PMI are sent back to the transmitter which shares the same precoding codebook table with the receiver. The actual precoding matrix $\mathbf{P} \in \mathbf{W}_{L}$ is a quantized release of $\mathbf{V}_{L}$, where $\mathbf{W}_{L}$ is the limited size codebook designed for $L$ layers transmission. Therefore, performance degradation is caused, and the CL is defined as follows [11]:

$$
C_{\text {loss }}=\left.C_{L}\right|_{\mathbf{P}=\mathbf{V}_{L}}-\left.C_{L}\right|_{\mathbf{P} \in \mathbf{W}_{L}} .
$$

The CL is affected by the receiver types and codebook design. In the high SNR region, ZF and MMSE receivers yield the same performance [20], and the influence of the limited size codebook on CL is dominated. Thereby, we focus on the theoretical derivation of CL in high SNR case. Let $\mathbf{S}=$ $\mathbf{P}^{\dagger} \mathbf{H}^{\dagger} \mathbf{H P}$; its $(i, j)$ th element can be easily written as

$$
\{\mathbf{S}\}_{i, j}=\mathbf{p}_{i}^{\dagger}\left(\sum_{k=1}^{N_{t}} \lambda_{k} \mathbf{v}_{k} \mathbf{v}_{k}^{\dagger}\right) \mathbf{p}_{j},
$$

where $\mathbf{p}_{i}$ and $\mathbf{p}_{j}$ represent the $i$ th and $j$ th columns of the precoding matrix $\mathbf{P}$.

In the following, we consider two special cases to derive succinct expressions of CL in the high SNR region.

(1) $L=1$ : in this case, there is only one complex element in S, and the SINR can be easily derived from (3):

$$
\operatorname{SINR}_{1}=\gamma_{0} \sum_{k=1}^{N_{t}} \lambda_{k}\left|\mathbf{p}_{1}^{\dagger} \mathbf{v}_{k}\right|^{2} .
$$

Substituting (4) and (12) into (10), we get the CL expression and an upper bound of single layer transmission:

$$
\begin{aligned}
\left.C_{\text {loss }}\right|_{L=1} & =-\log _{2} \sum_{k=1}^{N_{t}} \frac{\lambda_{k}}{\lambda_{1}}\left|\mathbf{p}_{1}^{\dagger} \mathbf{v}_{k}\right|^{2} \\
& \leq-\log _{2}\left|\mathbf{p}_{1}^{\dagger} \mathbf{v}_{1}\right|^{2} .
\end{aligned}
$$

(2) $L=N_{t}$ : in this case, the matrix $\mathbf{P}$ is full rank, and satisfying $\mathbf{P} \mathbf{P}^{\dagger}=\mathbf{P}^{\dagger} \mathbf{P}=\mathbf{I}_{N_{t}}$. According to the definition of inverse matrix, we have $\mathbf{S}^{-1}=\mathbf{P}^{\dagger} \mathbf{V} \boldsymbol{\Lambda}^{-1} \mathbf{V}^{\dagger} \mathbf{P}$. With the aid of (11), the SINR of the $l$ th layer can be represented as

$$
\operatorname{SINR}_{l}=\frac{\gamma_{0} / N_{t}}{\sum_{k=1}^{N_{t}} \frac{1}{\lambda_{k}}\left|\mathbf{p}_{l}^{\dagger} \mathbf{v}_{k}\right|^{2}}
$$

Similar to the case of $L=1$, invoking the property of concave function, we have

$$
\begin{aligned}
\left.C_{\text {loss }}\right|_{L=N_{t}} & =\sum_{l=1}^{N_{t}} \log _{2} \sum_{k=1}^{N_{t}} \frac{\lambda_{l}}{\lambda_{k}}\left|\mathbf{p}_{l}^{\dagger} \mathbf{v}_{k}\right|^{2} \\
& \leq N_{t} \log _{2} \frac{1}{N_{t}} \sum_{l=1}^{N_{t}} \sum_{k=1}^{N_{t}} \frac{\lambda_{l}}{\lambda_{k}}\left|\mathbf{p}_{l}^{\dagger} \mathbf{v}_{k}\right|^{2} .
\end{aligned}
$$


For open-loop spatial multiplexing (OLSM) transmission [21], no CSI is available at the transmitter. It is equivalent that the precoding matrix is unit, $\mathbf{P}=\mathbf{I}_{N_{t}}$. Substituting into (15), we get the CL upper bound of OLSM transmission:

$$
C_{\text {loss, } \mathrm{OL}} \leq N_{t} \log _{2} \frac{1}{N_{t}} \sum_{l=1}^{N_{t}} \sum_{k=1}^{N_{t}} \frac{\lambda_{l}}{\lambda_{k}}\left|\{\mathbf{V}\}_{l, k}\right|^{2} .
$$

2.3. Codebook Selection. Though a general form of CL is difficult to obtain, we still expect to utilize its compact expressions in two considered special cases to simplify the codebook selection from the perspective of CL minimization. In this paper, the following selection criterions are compared.

2.3.1. Maximum Capacity (MC). The precoding matrix $\mathbf{P}$ is selected from the codebook set $\mathbf{W}_{L}$ to maximize the capacity. The capacity of each codebook is calculated according to (3)(4). It can achieve the optimum performance at the price of high calculation complexity due to the matrix inversion.

2.3.2. Minimum Subspace Angle (MSA). An often used selection criterion is to minimize the Chordal distance [22] with much lower complexity than MC, expressed as

$$
\min _{\mathbf{P} \in \mathbf{W}_{L}}\left\{L-\sum_{l=1}^{L}\left|\mathbf{p}_{l}^{\dagger} \mathbf{v}_{l}\right|^{2}\right\} \text {. }
$$

2.3.3. Simplified MC. MC criterion is equivalent to minimizing CL in nature and can be simplified with the help of the compact CL expressions. From (13) and (15), a codebook selection criterion is directly obtained to minimize CL and organized as

$$
\begin{gathered}
\max _{\mathbf{P} \in \mathbf{W}_{L}}\left\{\sum_{k=1}^{N_{t}} \frac{\lambda_{k}}{\lambda_{1}}\left|\mathbf{p}_{1}^{\dagger} \mathbf{v}_{k}\right|^{2}\right\}, \quad L=1, \\
\min _{\mathbf{P} \in \mathbf{W}_{L}}\left\{\sum_{l=1}^{N_{t}} \sum_{k=1}^{N_{t}} \frac{\lambda_{l}}{\lambda_{k}}\left|\mathbf{p}_{l}^{\dagger} \mathbf{v}_{k}\right|^{2}\right\}, \quad L=N_{t} .
\end{gathered}
$$

2.4. MIMO-OFDM System Evaluation. The channel matrix of the $k$ th subcarrier is denoted as $\mathbf{H}^{k}, k=1,2, \ldots, K$, where $K$ is the total number of subcarriers. The corresponding capacity $C^{k}\left(L^{k}, \mathbf{P}^{k}\right)$ with $L^{k}$ layers and precoding matrix $\mathbf{P}^{k}$ can be calculated according to Section 2.1. The average spectral efficiency over the whole band is calculated as [20]

$$
C=\frac{1}{K} \sum_{k=1}^{K} C^{k}\left(L^{k}, \mathbf{P}^{k}\right)
$$

When the number of transmitted layers is fixed for all subcarriers and the precoding matrix of each subcarrier is optimally chosen from the corresponding codebook set, the capacity with $L$ layers transmission can be represented as

$$
C_{L}=\frac{1}{K} \sum_{k=1}^{K} \max _{\mathbf{P}^{k}} C^{k}\left(L, \mathbf{P}^{k}\right) .
$$

Due to the effect of frequency-selective fading, fixed layers transmission over the whole band is not the best strategy. The performance can be improved by layer adaption in frequency domain. Also the performance of FDLA will be upper bounded by the case that $L^{k}$ is optimized in subcarrier level:

$$
C_{\text {upper }}=\frac{1}{K} \sum_{k=1}^{K} \max _{L^{k}}\left\{\max _{\mathbf{P}^{k}} C^{k}\left(L^{k}, \mathbf{P}^{k}\right)\right\} .
$$

We define the maximum capacity boost of FDLA, $G_{\max }$, as the capacity difference between the capacity upper bound of FDLA and the capacity with optimum fixed layer transmission:

$$
G_{\max }=C_{\text {upper }}-\max _{L} C_{L} \geq 0 .
$$

The capacity improvement of FDLA depends on the channel fluctuation level in frequency domain. We first propose an effective metric to express the channel multiplexing capability then measure its fluctuation level. Generally, $K_{D}$ can be utilized to indicate the invertibility and full-rank multiplexing capability of the channel matrix [23]. However, it is not robust due to the dominated effect of the minimum channel eigenvalue; hence, its dynamic range in actual frequency-selective channel is very large (up to $40 \mathrm{~dB}$ or more) even if the channel yields favorable multiplexing capability across the whole band. More importantly, for MIMO systems with precoding at the transmitter, $K_{D}$ cannot comprehensively express the channel multilayer multiplexing capability. Here we define a new channel multiplexing capability metric $\mathcal{M}$ as follows:

$$
\mathcal{M}=\frac{\|\mathbf{H}\|_{F}^{2}}{\|\mathbf{H}\|_{F}^{2}-\lambda_{\max }},
$$

where the numerator equals to the sum of the channel eigenvalues. The relationship between $\mathcal{M}$ and the channel condition number is

$$
\mathcal{M} \leq \frac{K_{D}}{N_{t}-1} \leq \frac{N_{t}}{N_{t}-1} \frac{\lambda_{\max }}{\lambda_{\min }} .
$$

Substituting (23) into (6), an equivalent expression is derived:

$$
\mathcal{M} \geq \frac{\gamma_{0}\|\mathbf{H}\|_{F}^{2}}{N_{t}\left(N_{t}-1\right)\left(N_{t}^{1 /\left(N_{t}-1\right)}-1\right)} .
$$

In the following, we define function $F(\zeta)$ to measure the channel fluctuation level within a subband:

$$
F(\zeta)=\frac{\zeta \sum_{i=1}^{\zeta} x_{i}^{2}}{\left(\sum_{i=1}^{\zeta} x_{i}\right)^{2}}-1,
$$

where $\zeta$ is the number of the subcarriers within the subband, and $x_{i}$ is the channel metric of the $i$ th subcarrier belonging to the considered subband. $x_{i}$ can be one element of the channel metric set $\left\{\|\mathbf{H}\|_{F}^{2}, \lambda_{\max }, \mathcal{M}\right\}$, where $\|\mathbf{H}\|_{F}^{2}$ is related to the received SNR and the performance of space time block codes $[24,25]$, and $\lambda_{\max }$ is the crucial parameter of MIMO beamforming scheme [26]. As $\zeta \rightarrow+\infty, F(\zeta)$ is actually the ratio between the variance and squared mean value of a random variable. 

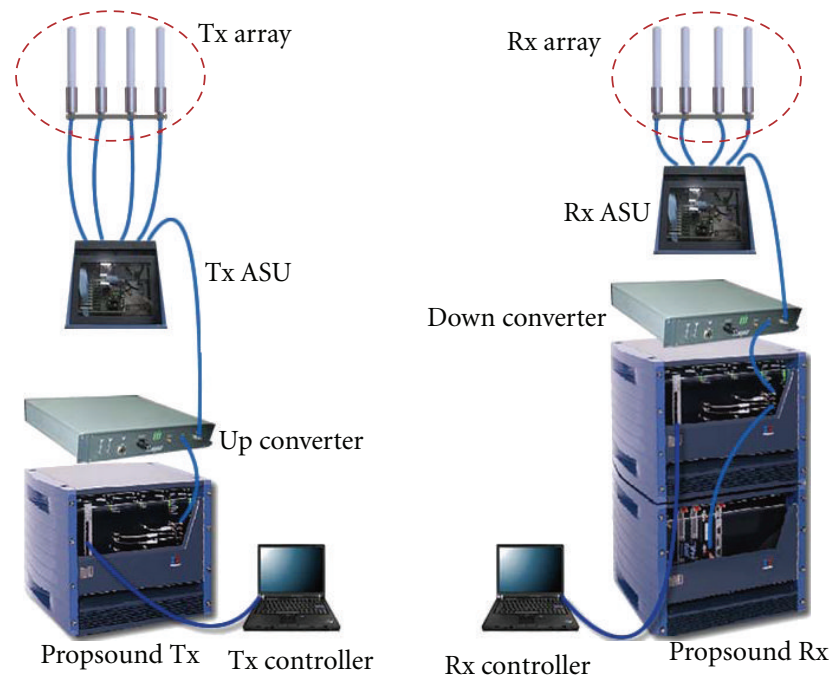

Figure 1: The setup of the MIMO channel measurement system.

\section{Measurement Description}

3.1. Measurement Equipment. Measurement was performed in a teaching building of Beijing University of Posts and Telecommunications utilizing the Elektrobit PropSound Channel Sounder [27] system illustrated in Figure 1. External RF conversion modules are deployed at both transmit and receive sides to support the operating frequency $6 \mathrm{GHz}$. Uniform linear array (ULA) with four dipoles has been equipped at both sides, which can be replaced by omnidirectional array (ODA) to extract spatial angle parameters of multipaths. One complete set of MIMO channel realization called cycle is captured in a time-division multiplexing (TDM) method. The measurement of each antenna pairs is accomplished with the help of the high-speed antenna switching unit (ASU) to transfer the antennas in sequence. Before the measurement, a back-to-back test is required to obtain the system response for calibration purpose, where the transmitter and receiver are connected directly by cable using a $50 \mathrm{~dB}$ attenuator to prevent power overload at the receiver.

3.2. Measurement Environment. The measurement was conducted in a typical indoor hall of Beijing University of Posts and Telecommunications. The layout and measurement position arrangement are shown in Figure 2. The red dots represent the measurement positions, also the measurement position index and moving direction (blue arrow lines) are marked out. The height of the transmit antenna array is $3 \mathrm{~m}$ and marked by black pentacle. The transmit array remained stationary in the center of the hall during the experiment. The channel is sampled in a fixed-position method. The receiver moved to the next measurement position once more than 700 sets of channel realizations are collected. Total number of measurement positions is 36 , and the lineof-sight (LOS) propagation component is always existent. The $x$ th measurement position is denoted as Pos. $x$. The separation between two adjacent measurement positions is $1.6 \mathrm{~m}$ (32 wavelengths). Reflect and scatter components

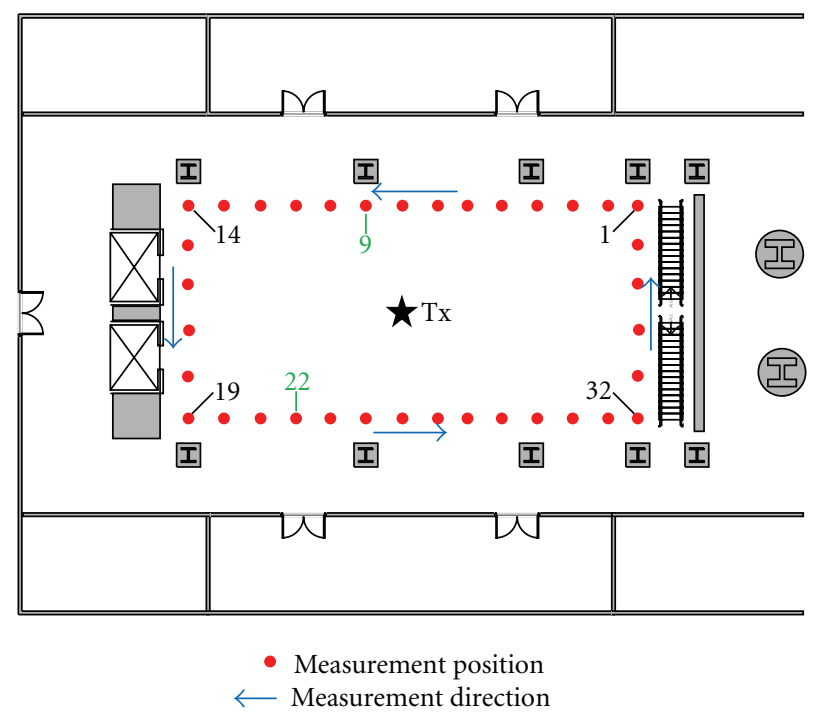

FIGURE 2: Indoor channel measurement layout and measurement position arrangement.

TABLE 1: Measurement configuration.

\begin{tabular}{lc}
\hline Items & Settings \\
\hline Center frequency $(\mathrm{GHz})$ & 6 \\
Bandwidth (MHz) & 100 \\
PN code length (chips) & 255 \\
Type of antenna array & ULA \\
Type of polarization & Vertical \\
Number of transmit antenna & 4 \\
Number of receive antenna & 4 \\
Element space of Tx $(\lambda)$ & 1 \\
Element space of $\mathrm{Rx}(\lambda)$ & 0.5 \\
Height of Tx antenna $(\mathrm{m})$ & 3 \\
Height of Rx antenna $(\mathrm{m})$ & 1.8 \\
\hline
\end{tabular}

are created by surrounding concrete wall, square columns $(1.2 \mathrm{~m} \times 1.2 \mathrm{~m})$, evaluators, stairs, and people. Total number of channel realizations is over $10^{5}$. The detailed measurement configuration is listed in Table 1.

Although we have evaluated the MIMO channels of all measurement positions, it is convenient to pick out some typical measurement positions to address the key problems. We use the proposed metric $\mathcal{M}$ to distinguish different channel conditions. Hence, Pos.9 and Pos.22 with high and low $\mathcal{M}$ are selected and marked by green color in Figure 2, whose $\mathcal{M}$ are the upper and lower bounds of our measurement campaign, respectively. We use Case I and Case II to represent Pos.9 and Pos.22, respectively, and the cumulative probability curves of $\mathcal{M}$ are plotted in Figure 3.

\section{Simulation Results}

Based on the indoor MIMO channel measurement and data after processing, we present the simulation results in this section. We select the closed-loop spatial multiplexing 


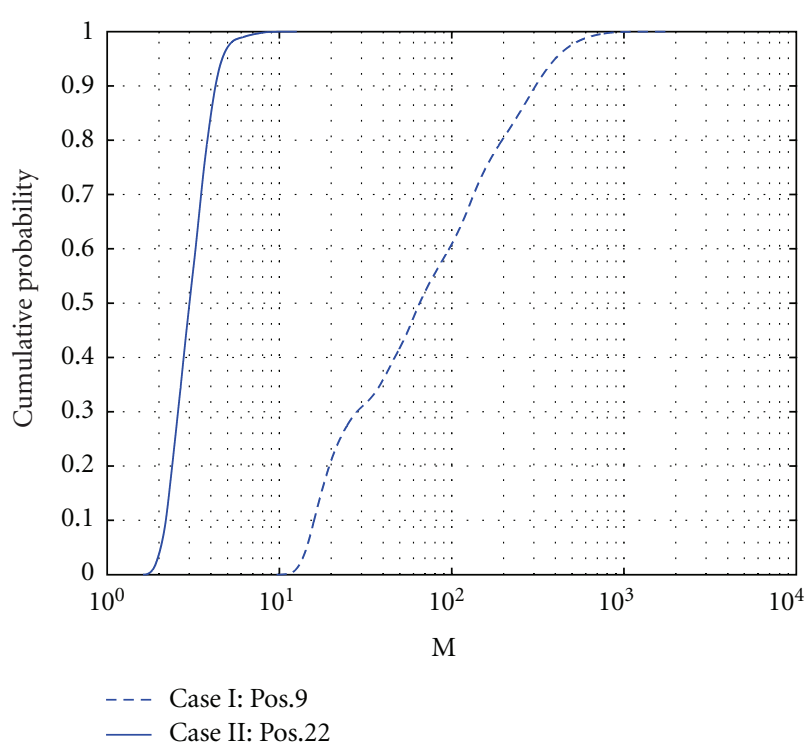

FIGURE 3: Cumulative probability of $\mathcal{M}$ in two considered cases.

(CLSM) codebook [6] standardized in LTE for evaluation, though the theoretical derivation in Section 2 is not limited by the codebook type. Each realization of the MIMO channel is transformed into the frequency domain by FFT, and the bandwidth of each subcarrier is $50 \mathrm{KHz}$. No power control strategy is adopted, and we consider single-user MIMO here. The symbols L1, L2, L3, and L4 represent the fixed layers transmission with one, two, three, and four layers, respectively.

4.1. Capacity with Perfect Precoding. Though unrealistic in practice, we wish to study the capacity with perfect precoding to provide the performance limit under different channel conditions. The upper bounds represent the maximum capacity of FDLA mentioned in (21). It can be found from Figure 4 that when the SNR is lower than $2 \mathrm{~dB}$ in Case I, $G_{\max }=C_{\text {upper }}-C_{1} \rightarrow 0$, which indicates that single layer transmission is nearly optimal for all subcarrier and no benefit is obtained by FDLA. Similar phenomenon is observed in Case II when the SNR is higher than $23 \mathrm{~dB}$. Moreover, the maximum capacity boost of FDLA is very limited and lower than $0.8 \mathrm{bit} / \mathrm{s} / \mathrm{Hz}$ for all considered SNR under different channel conditions. The crossing point between the capacity curves of different layers transmission is greatly influenced by channel condition as indicated in (25), and the crossing point between $\mathrm{L} 1$ and $\mathrm{L} 4$ is about $13 \mathrm{~dB}$ and $0 \mathrm{~dB}$ in Case I and Case II, respectively.

4.2. Capacity with CLSM Codebook. The use of limited size codebook will bring interlayer interference and lead to performance degradation. We first compare the performance of different layers transmission with ZF and MMSE receivers and then evaluate the CL due to the limited feedback. From Figure 5, L1 transmissions with ZF and MMSE receivers yield the same performance over the whole SNR range, which can be easily proved through the SINR expressions (3). For multi-layer transmission, the performance of ZF and MMSE receivers will converge when the SNR is large enough. In Case I with high $\mathcal{M}$, the performance of MMSE receiver greatly outperforms ZF receiver in low and median SNR regions when $L \geq 2$. It indicates that the effect of noise is leading compared to the interlayer interference and suppressed by MMSE receiver which is equivalent to a matched filter in the low SNR region. In Case II with low $\mathcal{M}, \mathrm{ZF}$ and MMSE receivers yield identical performance within the considered SNR scope when $L<4$, which reveals that the influence of the interlayer interference is dominant. The performance difference between two type receivers of L4 transmission in case II is led by the layer with the worst SINR. In conclusion, the performance of ZF and MMSE receivers will converge quickly within practical SNR range when $L<4$, but the full-rank L4 transmission yields a much slower convergence speed. An interesting finding is that the FDLA upper bounds of ZF and MMSE receivers are approximately identical for any given SNR. Furthermore, the maximum capacity boost $G_{\max }$ of FDLA is especially significant in case of high $\mathcal{M}$.

Since the performance of MMSE receiver greatly exceeds ZF receiver in low SNR range, we expect to study the CL of different layers transmission with MMSE receiver. Figure 6 shows that L1 transmission suffers very slight capacity loss over the whole SNR scope, which indicates that current codebook design for L1 transmission is nearly capacity lossless. The main reason is that there is no interlayer interference, and the limited size codebook only causes a little beamforming direction bias which leads to a slight SNR gain decrease. As the number of transmitted layers increasing, the CL becomes more significant and especially severe for L4 transmission. It reveals that the limited size codebook set cannot simultaneously offer accurate beamforming vectors for multi-layer transmission to eliminate the interlayer interference and the situation gets worse when the transmitted layers increase. Moreover, the CL in high $\mathcal{M}$ case is larger than that of low $\mathcal{M}$ case, which can be roughly explained by the CL expression of full-rank transmission derived in (15). The percentage CLs of L1, L2, L3, and L4 transmissions relative to perfect precoding at $25 \mathrm{~dB}$ is respectively $5.5 \%$, $21 \%, 30 \%$, and $39 \%$ in Case I, corresponding to $3.8 \%, 6.7 \%$, $8.2 \%$, and $19.5 \%$ in Case II. Due to the CL difference, the crossing points between different layers transmissions are greatly changed compared to the perfect precoding case. For instance, the crossing points between L1 and L4 transmissions are, respectively, $27 \mathrm{~dB}$ and $2 \mathrm{~dB}$ in Case I and Case II.

4.3. Codebook Selection. Performance comparisons between the considered codebook selection criterions are given in Figure 7. For L1 transmission, MSA criterion is equivalent to the minimization of the CL upper bound derived in (13), and the MC and simplified MC criterions are essentially the same. All three selection criterions yield identical performance. Benefitting from taking the interlayer interference into account, the MC and simplified MC criterions outperform the MSA criterion for L4 transmission. Because the simplified MC criterion for L4 transmission is based on the CL upper bound in (15), performance decrease relative to MC 


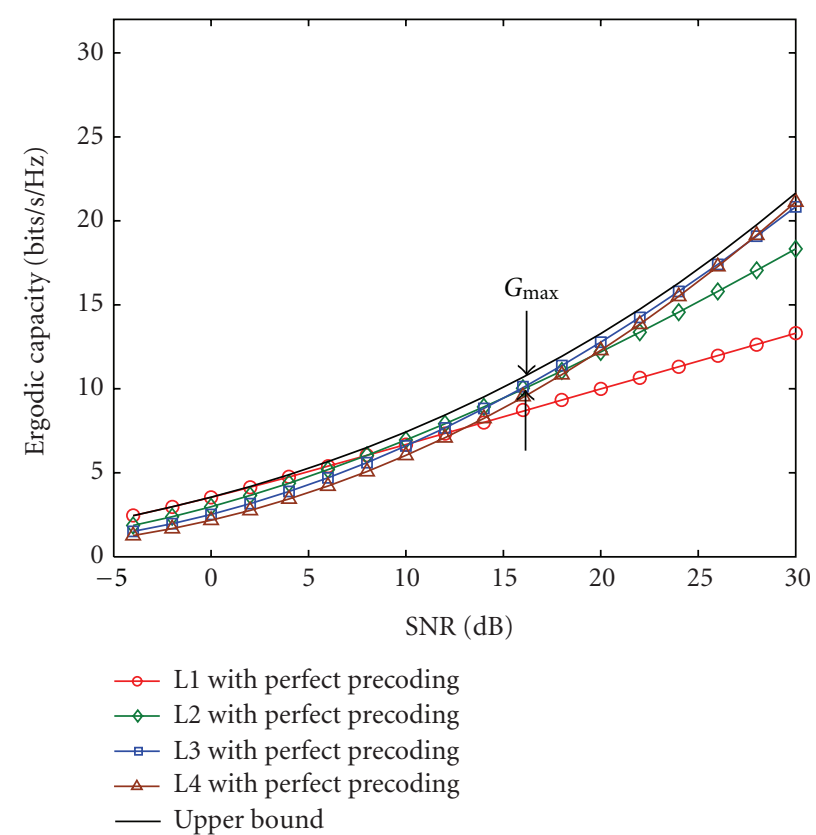

(a) Case I: high $\mathcal{M}$

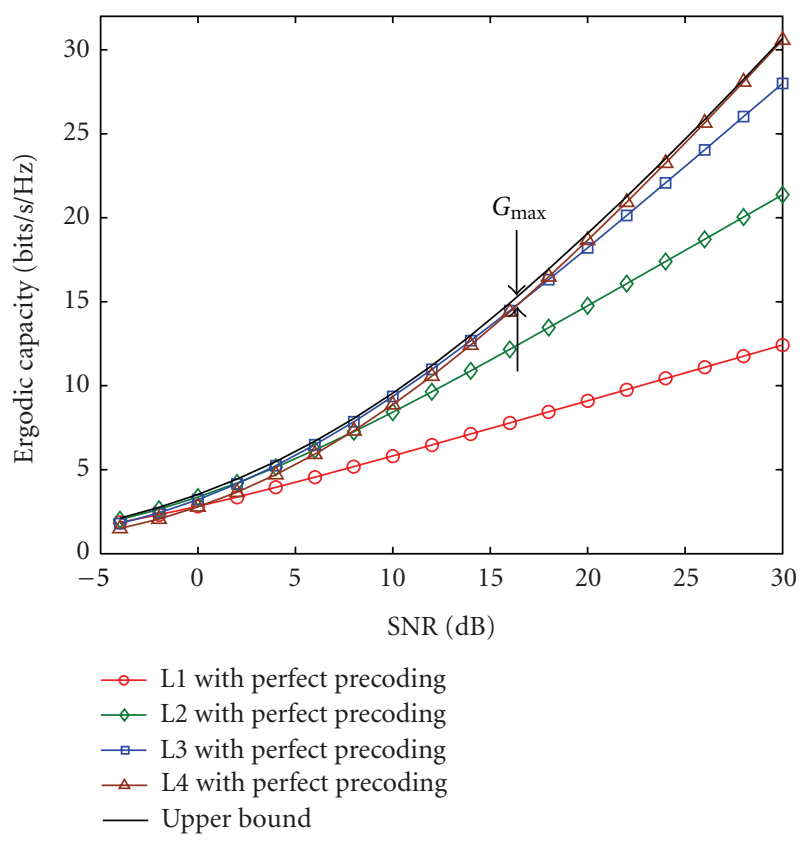

(b) Case II: low $\mathcal{M}$

FIGURE 4: Capacity of different layer transmissions with perfect precoding.

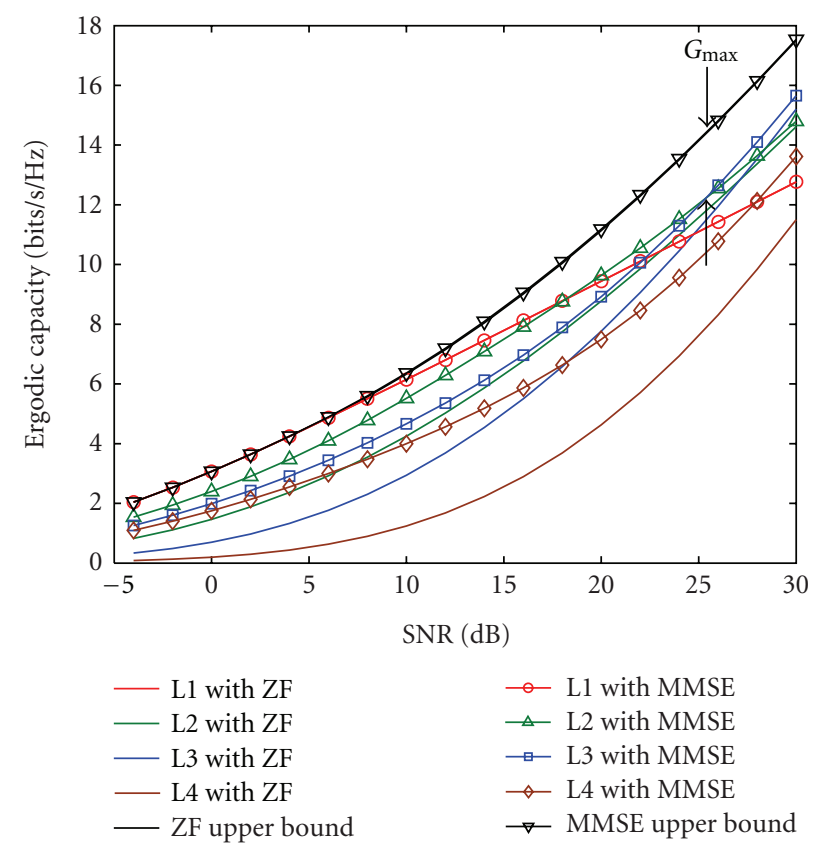

(a) Case I: high $\mathcal{M}$

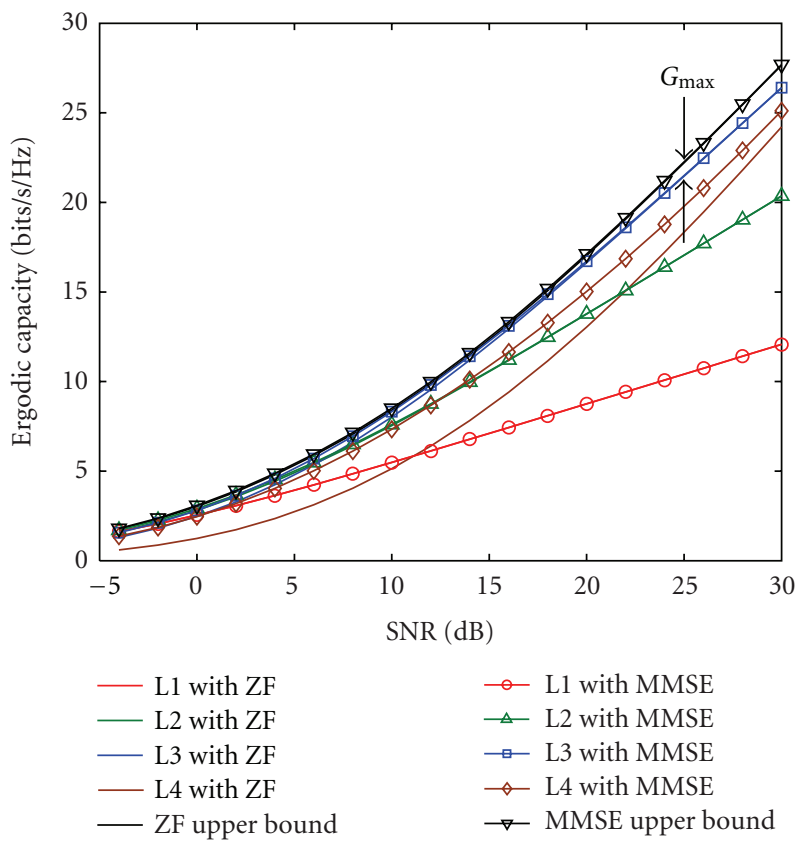

(b) Case II: low $\mathcal{M}$

Figure 5: Capacity of different layer transmissions with ZF and MMSE receivers using CLSM codebook.

criterion is resulted. However, compared to the CL marked in Figure 6, the performance improvement by the use of better codebook selection criterions is very limited. To approve that, capacity comparison between CLSM and OLSM with L4 transmission is also plotted in Figure 8. In Case I, the capacity improvement of CLSM compared to OLSM is $1.72 \mathrm{bit} / \mathrm{s} / \mathrm{Hz}$ at $30 \mathrm{~dB}$; yet the $\mathrm{CL}$ due to the limited size CLSM codebook reaches $7.5 \mathrm{bit} / \mathrm{s} / \mathrm{Hz}$. Though considering single user here, it must be mentioned that the simplified MC criterion for fullrank transmission can also be used in multiuser MIMO to reduce the interference.

4.4. Effects of Channel Variation. In our measurement campaign, the transmitter and receiver are stationary during the 


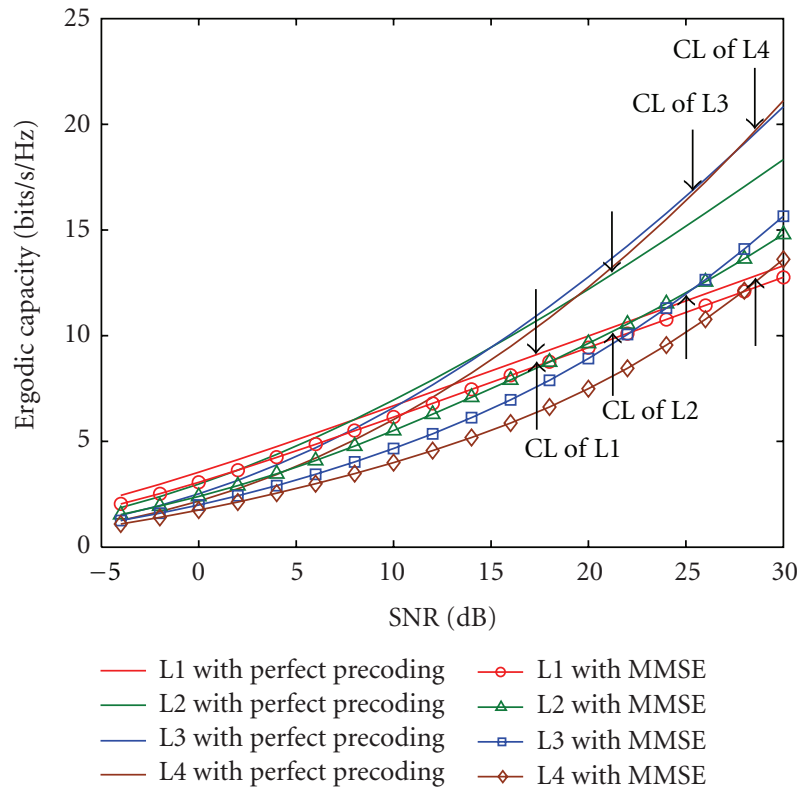

(a) Case I: high $\mathcal{M}$

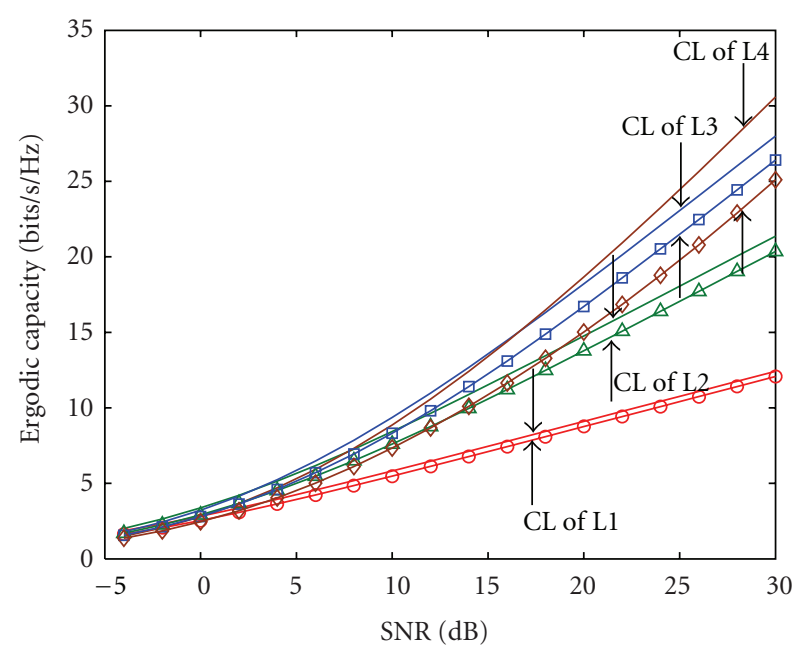

$\begin{array}{ll}\text { — L1 with perfect precoding } & \rightarrow \text { L1 with MMSE } \\ \text { - L2 with perfect precoding } & - \text { L2 with MMSE } \\ \text { — L3 with perfect precoding } & \rightarrow \text { L3 with MMSE } \\ \text { - L4 with perfect precoding } & \rightarrow \text { L4 with MMSE }\end{array}$

(b) Case II: low $\mathcal{M}$

FIGURE 6: CL comparison between different layer transmissions with MMSE receiver using CLSM codebook.

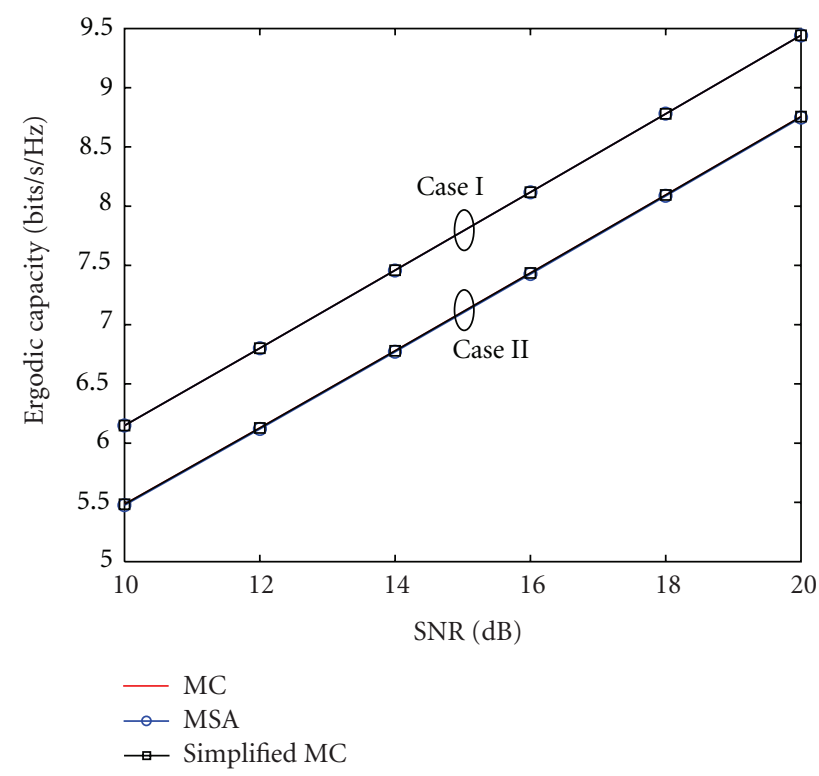

(a) L1 transmission

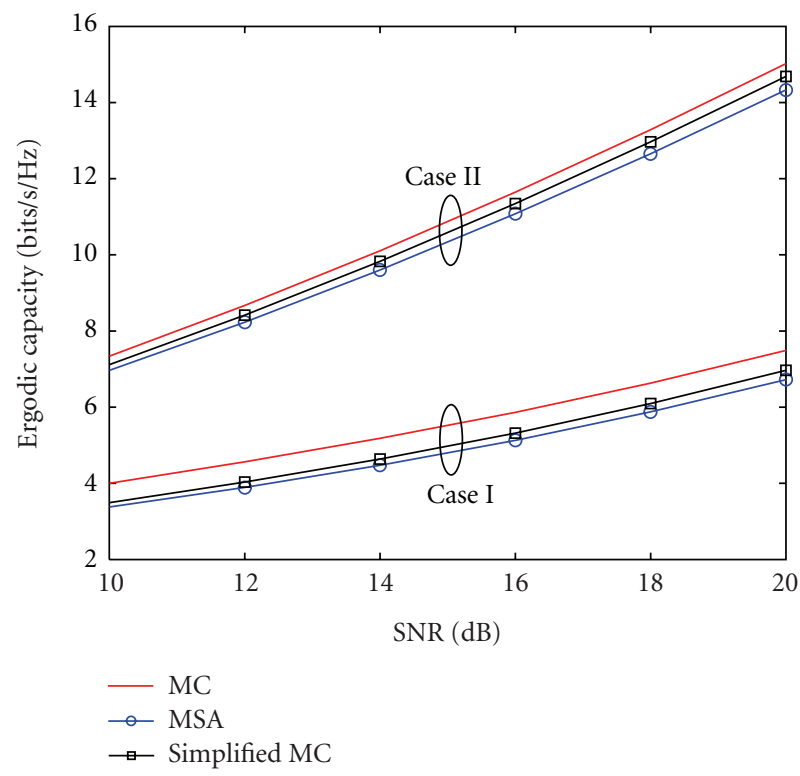

(b) L4 transmission

Figure 7: Performance comparison between different codebook selection criterions for L1 and L4 transmissions with MMSE receiver.

channel sampling process, so we only consider the effect of frequency domain channel variation on the performance of MIMO-OFDM system here. The rich reflect and scatter objects in indoor environment result in channel frequency selective feature, thus expanding the available adaption dimension in frequency domain. The performance of FDLA is closely related to the channel fluctuation level within a subband. Using the function $F(\zeta)$ defined in (26), we first evaluate the key channel metrics $\left\{\|\mathbf{H}\|_{F}^{2}, \lambda_{\max }, \mathcal{M}\right\}$ fluctuation level within a subband. Then the influence of the frequency domain feedback interval on the performance of FDLA is investigated, which is helpful to achieve the tradeoff between the performance degradation and amount of feedback bits.

From Figure 9, the fluctuation level variations of $\|\mathbf{H}\|_{F}^{2}$ and $\lambda_{\max }$ are highly coherent, and an intuitive explanation is that the maximum eigenvalue dominates the power sum of all the layers. The fluctuation level of $\mathcal{M}$ seems to be related 


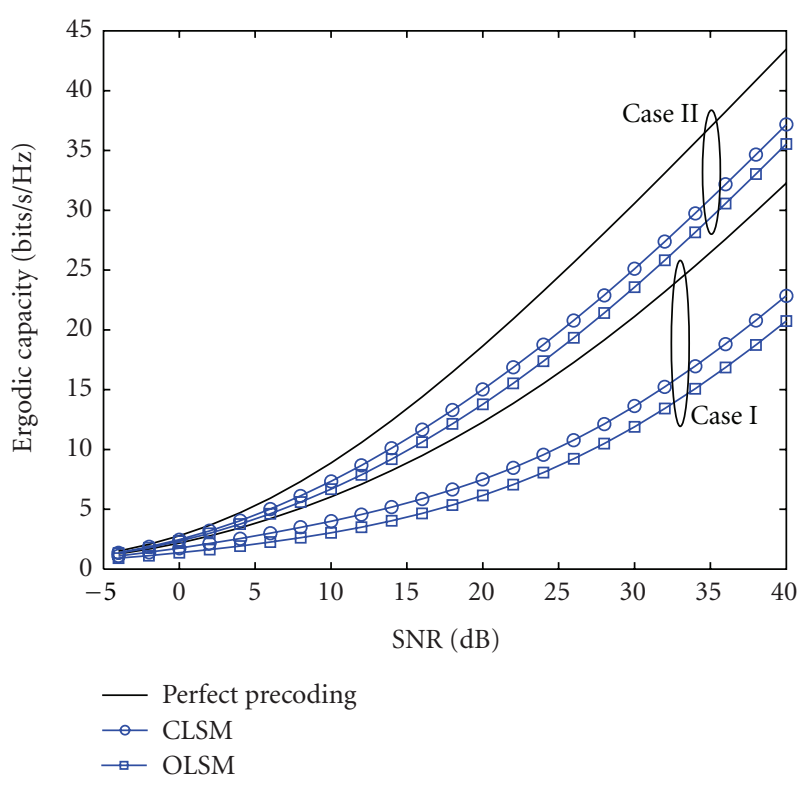

Figure 8: Capacity comparison between CLSM and OLSM for L4 transmission with MMSE receiver.

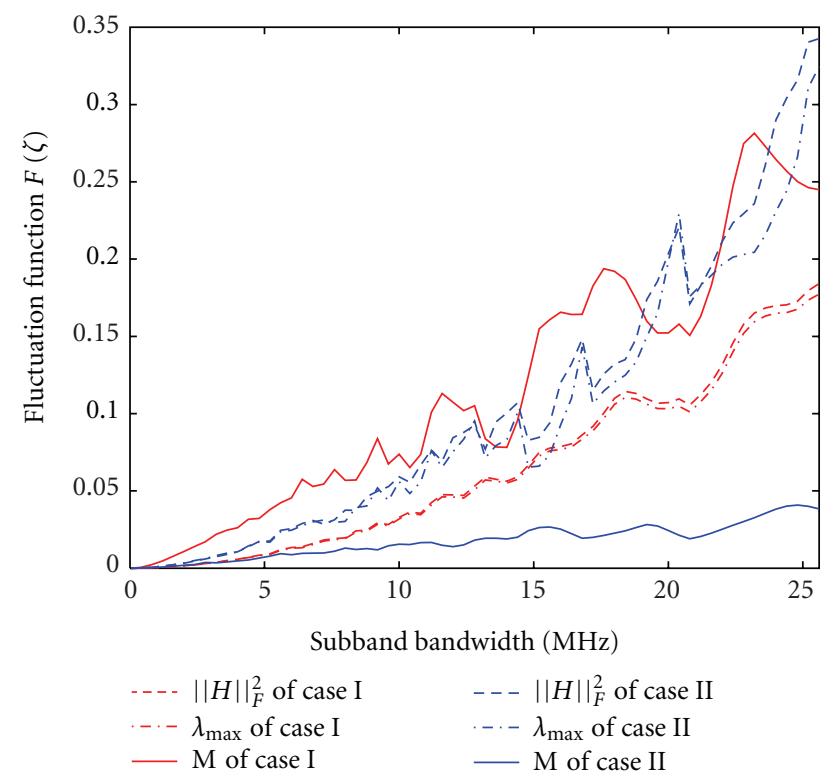

Figure 9: The fluctuation level variations of the key channel metrics.

to the value of $\mathcal{M}$ itself. In Case I with high $\mathcal{M}$, the fluctuation of $\mathcal{M}$ is severer than $\|\mathbf{H}\|_{F}^{2}$ and $\lambda_{\max }$. In contrast, the $\mathcal{M}$ varies much smoother within a subband in Case II.

In Figure 10, the percentage capacity is plotted, which is normalized by the FDLA upper bound $C_{\text {upper }}$ in (21). A capacity floor is resulted as the transmitted layers feedback interval increasing, also the descend speed and value of the floor are related to the fluctuation level of the multiplexing capability metric $\mathcal{M}$. The higher the fluctuation level of $\mathrm{M}$, the lower the value of the capacity floor. The maximum

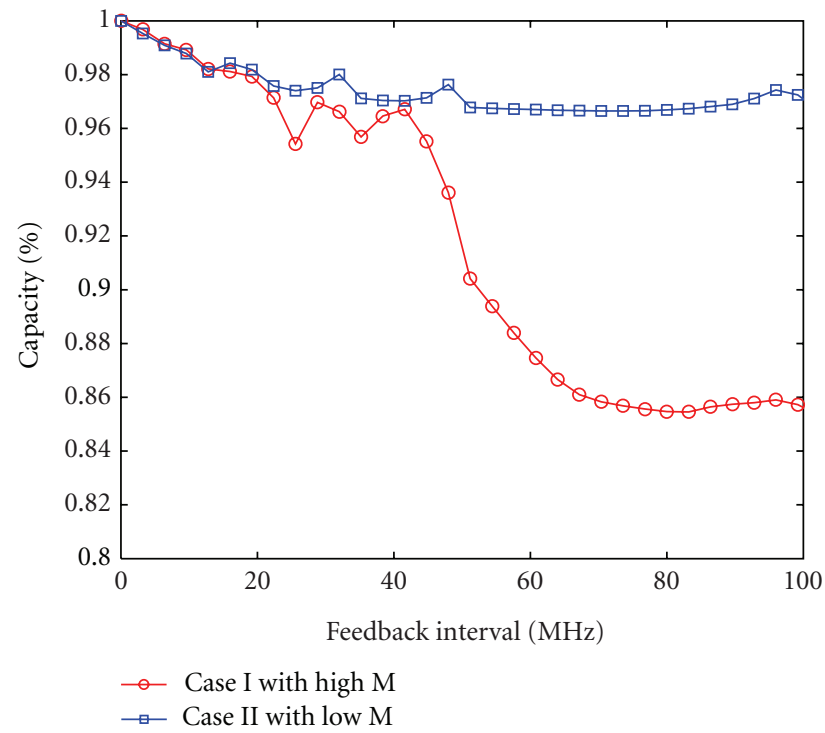

FIgure 10: Percentage capacity variation as the transmitted layers feedback interval in frequency domain.

capacity degradation is about $3 \%$ and $14 \%$, respectively, in two considered cases.

\section{Conclusion}

Based on the wideband MIMO channel measurement in a typical indoor scenario, this paper has evaluated the performance of LTE/LTE-A CLSM codebook and the effect of channel variation on MIMO-OFDM system. Under the perfect precoding assumption, very slight capacity gain is obtained by FDLA. However, considering the limited size codebook, the maximum capacity boost of FDLA is highly related to the SNR and channel conditions. It is interesting to find that the FDLA upper bounds of ZF and MMSE receivers are identical under different channel conditions. The results show that current codebook design for L1 transmission is nearly capacity lossless, and the CL increases with the number of transmitted layers. Generally, the CL in high $\mathcal{M}$ case is more significant than that in low $\mathcal{M}$ case. Furthermore, compared to the CL caused by the limited size codebook, the capacity improvement of better codebook selection criterions is very limited. It reveals that the fluctuation levels of $\|\mathbf{H}\|_{F}^{2}$ and $\lambda_{\max }$ within a subband are interrelated, and the fluctuation level of $\mathcal{M}$ is determined by the value of $\mathcal{M}$ itself. Finally, a capacity floor is resulted as the layer number feedback interval increasing, and the descending speed and value of the floor are greatly influenced by the fluctuation level of $\mathcal{M}$.

\section{Acknowledgments}

The research is supported in part by China Important National Science and Technology Specific Projects under Grant no. 2012ZX03001043-009 and by Telecommunications Science Technical Institute (Datang Telecommunication Technology and Industry Group). 


\section{References}

[1] M. El-Hajjar and L. Hanzo, "Multifunctional mimo systems: a combined diversity and multiplexing design perspective," IEEE Wireless Communications, vol. 17, no. 2, pp. 73-79, 2010.

[2] C. S. Park, Y. P. E. Wang, G. Jöngren, and D. Hammarwall, "Evolution of uplink MIMO for LTE-advanced," IEEE Communications Magazine, vol. 49, no. 2, pp. 112-121, 2011.

[3] L. Liu, R. Chen, S. Geirhofer, K. Sayana, Z. Shi, and Y. Zhou, "Downlink MIMO in LTE-advanced: SU-MIMO versus MUMIMO," IEEE Communications Magazine, vol. 50, no. 2, pp. 140-147, 2012.

[4] F. Boccardi, B. Clerckx, A. Ghosh et al., "Multiple-antenna techniques in LTE-advanced," IEEE Communications Magazine, vol. 50, no. 3, pp. 114-121, 2012.

[5] D. J. Love, R. W. Heath, V. K. N. Lau, D. Gesbert, B. D. Rao, and M. Andrews, "An overview of limited feedback in wireless communication systems," IEEE Journal on Selected Areas in Communications, vol. 26, no. 8, pp. 1341-1365, 2008.

[6] 3GPP TS 36.211, "Evolved universal terrestrial radio access (EUTRA), physical channels and modulation," vol. V10.4.0, 2012.

[7] W. Santipach and M. L. Honig, "Capacity of a multipleantenna fading channel with a quantized precoding matrix," IEEE Transactions on Information Theory, vol. 55, no. 3, pp. 1218-1234, 2009.

[8] R. de Miguel, V. Gardašević, R. R. Müller, and F. F. Knudsen, "On overloaded vector precoding for single-user MIMO channels," IEEE Transactions on Wireless Communications, vol. 9, no. 2, pp. 745-753, 2010.

[9] P. Xiao, R. Tafazolli, K. Moessner, and A. Gluhak, "Codebook based single-user MIMO system design with widely linear processing," IEEE Transactions on Communications, vol. 60, no. 9, pp. 2408-2414, 2012.

[10] H. J. Yang, J. Chun, Y. Choi, S. Kim, and A. Paulraj, "Codebook-based lattice-reduction-aided precoding for limitedfeedback coded MIMO systems," IEEE Transactions on Communications, vol. 60, no. 2, pp. 510-524, 2012.

[11] M. Zhang, M. Shafi, P. J. Smith, and P. A. Dmochowski, "Precoding performance with codebook feedback in a MIMOOFDM system," in Proceedings of the IEEE International Conference on Communications (ICC'11), pp. 1-6, June 2011.

[12] W. Kim, H. Lee, M. Kim, and H. Chung, "Distribution of eigenvalues for $2 \times 2$ MIMO channel capacity based on indoor measurements," IEEE Transactions on Wireless Communications, vol. 11, no. 4, pp. 1255-1259, 2012.

[13] C. Oestges and N. Czink, "Empirical investigation of multilink separation for indoor MIMO channels," in Proceedings of the IEEE International Symposium on Personal, Indoor and Mobile Radio Communications (PIMRC '11), pp. 945-949, September 2011.

[14] J. Poutanen, J. Salmi, K. Haneda, V. M. Kolmonen, and P. Vainikainen, "Angular and shadowing characteristics of dense multipath components in indoor radio channels," IEEE Transactions on Antennas and Propagation, vol. 59, no. 1, pp. 245253, 2011.

[15] C. Mehlführer, S. Caban, and M. Rupp, "Measurement-based performance evaluation of MIMO HSDPA," IEEE Transactions on Vehicular Technology, vol. 59, no. 9, pp. 4354-4367, 2010.

[16] Y. Ofuji, H. Takahashi, S. Fukumoto, S. Abeta, and T. Nakamura, "Field experimental results of E-UTRA downlink with precoding and non-precoding MIMO," in Proceedings of the IEEE 69th Vehicular Technology Conference, pp. 1-5, April 2009.
[17] K. P. Kongara, P. J. Smith, and L. M. Garth, "Frequency domain variation of eigenvalues in adaptive MIMO OFDM systems," IEEE Transactions on Wireless Communications, vol. 10, no. 11, pp. 3656-3665, 2011.

[18] D. Dong, J. Zhang, Y. Zhang, and X. Nie, "Large scale characteristics and capacity evaluation of outdoor relay channels at $2.35 \mathrm{GHz}$," in Proceedings of the IEEE 70th Vehicular Technology Conference Fall (VTC’ 09), September 2009.

[19] R. W. Heath Jr., S. Sandhu, and A. Paulraj, "Antenna selection for spatial multiplexing systems with linear receivers," IEEE Communications Letters, vol. 5, no. 4, pp. 142-144, 2001.

[20] D. Tse and P. Viswanath, Fundamentals of Wireless Communication, Cambridge University Press, New York, NY, USA, 2005.

[21] A. Forenza, M. R. McKay, A. Pandharipande, R. W. Heath, and I. B. Collings, "Adaptive MIMO transmission for exploiting the capacity of spatially correlated channels," IEEE Transactions on Vehicular Technology, vol. 56, no. 2, pp. 619-630, 2007.

[22] N. Ravindran and N. Jindal, "Limited feedback-based block diagonalization for the MIMO broadcast channel," IEEE Journal on Selected Areas in Communications, vol. 26, no. 8, pp. 1473-1482, 2008.

[23] R. W. Heath and A. J. Paulraj, "Switching between diversity and multiplexing in MIMO systems," IEEE Transactions on Communications, vol. 53, no. 6, pp. 962-968, 2005.

[24] J. Huang and S. Signell, "Adaptive MIMO systems in $2 \times 2$ uncorrelated Rayleigh fading channel," in Proceedings of the IEEE Wireless Communications and Networking Conference (WCNC'07), pp. 1114-1118, March 2007.

[25] J. Huang and S. Signell, "On spectral efficiency of lowcomplexity adaptive MIMO systems in Rayleigh fading channel," IEEE Transactions on Wireless Communications, vol. 8, no. 9, pp. 4369-4374, 2009.

[26] K. P. Kongara, P. H. Kuo, P. J. Smith, L. M. Garth, and A. Clark, "Performance analysis of adaptive MIMO OFDM beamforming systems," in Proceedings of the IEEE International Conference on Communications (ICC'08), pp. 4359-4365, May 2008.

[27] Elektrobit, "Propsoundmultidimensional channel sounder," http://www.elektrobit.com/. 

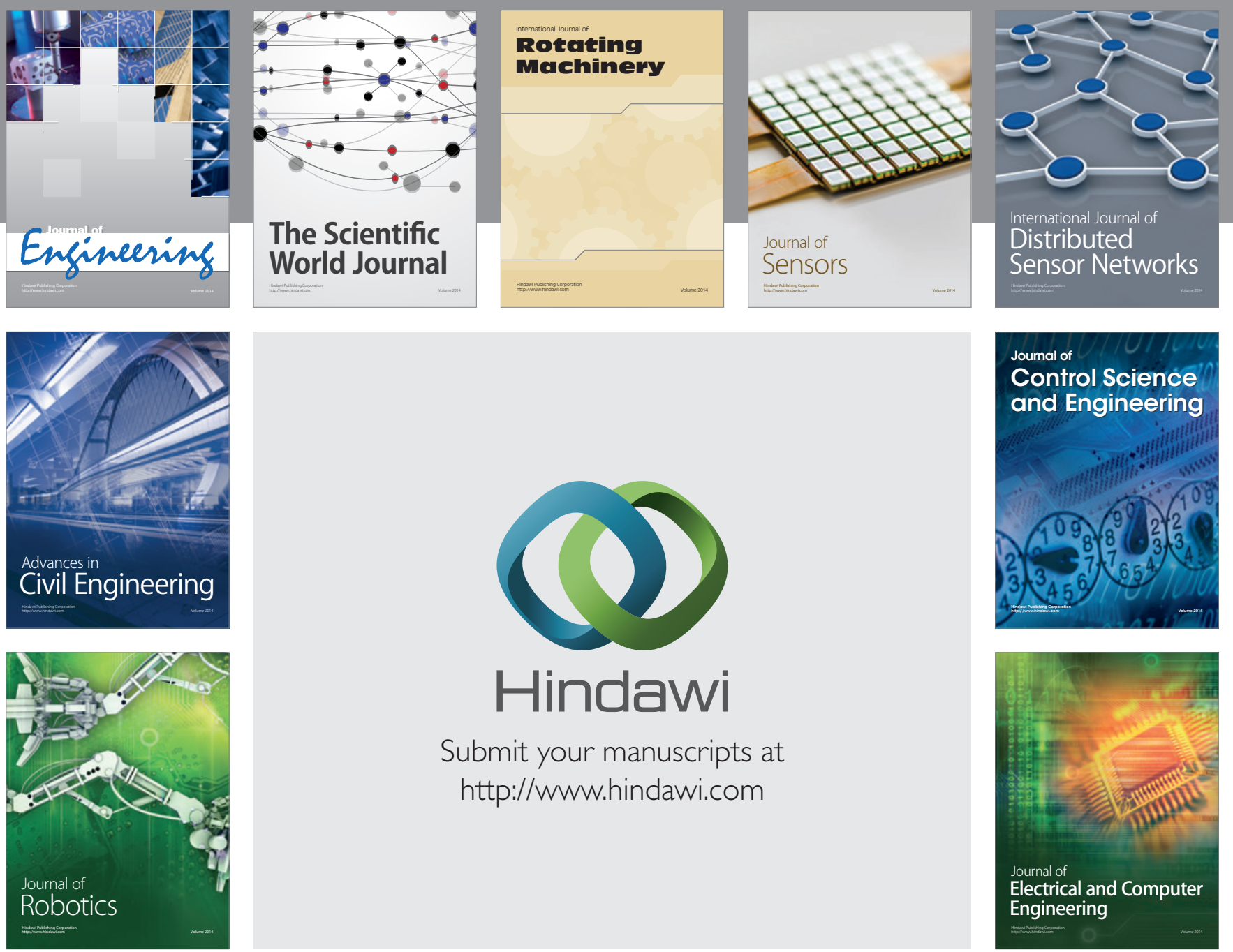

Submit your manuscripts at

http://www.hindawi.com
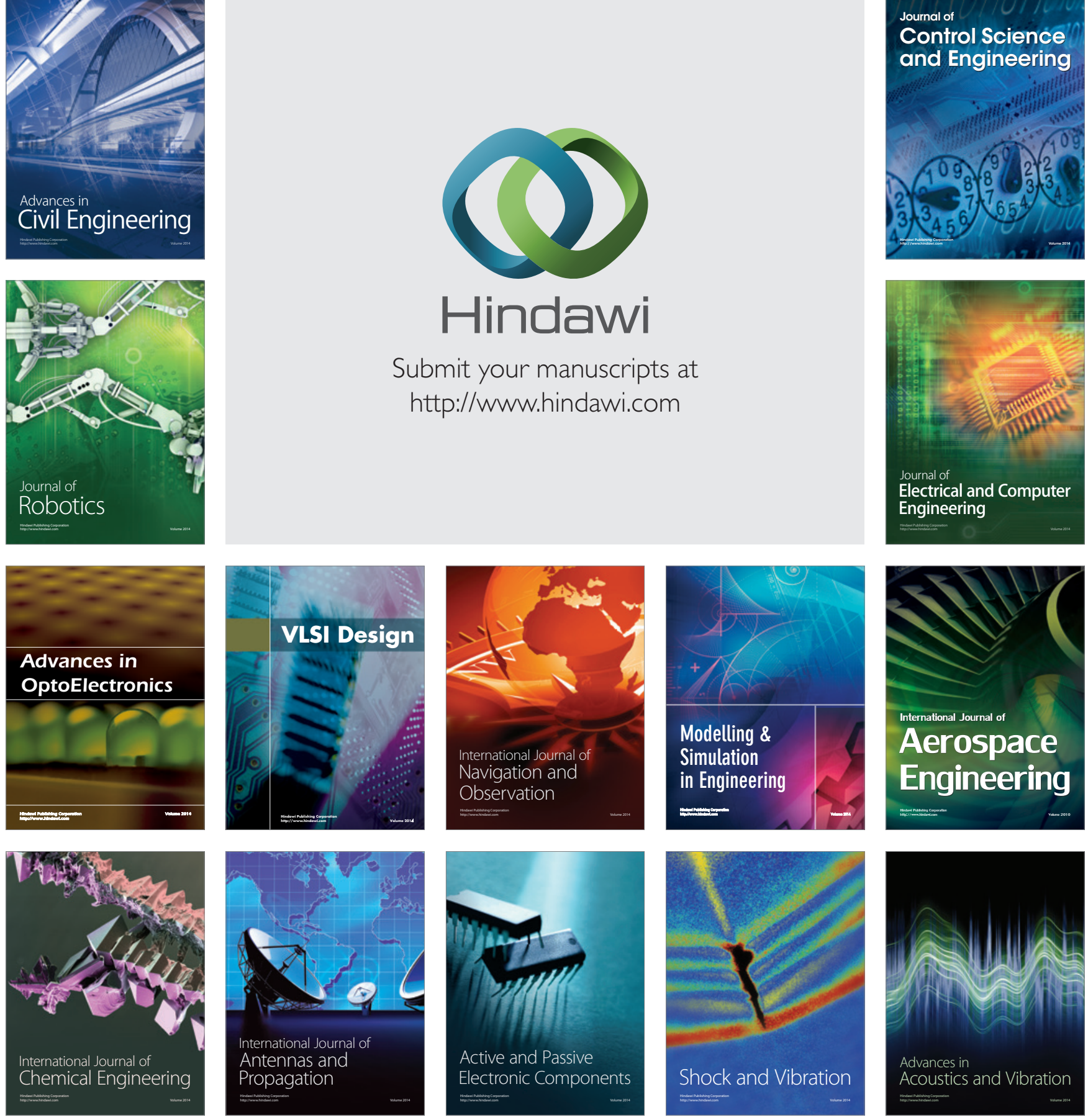